\title{
STUDY ON CHARACTERISTICS OF A DOWNHOLE VORTEX JET PUMP
}

\section{Denis Panevnyk}

Ivano-Frankivsk National Technical University of Oil and Gas, Ukraine

e-mail: den.panevnik@gmail.com

DOI: $10.51865 / J P G T .2021 .02 .03$

\begin{abstract}
This article analyzes the possibility of increasing the efficiency of using downhole jet pumps by swirling the injected flow. To analyze the peculiarities of the local swirling of injected flow, design and technological parameters in the form of the inclination angle of guiding elements, the diameter of the helical trajectory described by the fluid particles, and the flow rates of the swirling flow are studied. Based on the application of the conservation law of fluid momentum in adjacent jets with a parabolic pressure distribution, equations to determine the pressure characteristic of a jet pump are obtained, taking into account the additional dynamic pressure made by swirling the injected flow. In the process of analyzing the obtained relations, has been set the dependence of the relative pressure growth and the efficiency of the ejection system under the conditions of injected flow swirling on the relative flow rate of a jet pump, and an inversely proportional dependence of the above parameters on its main geometric parameter in the form of the ratio of cross-sectional areas of the mixing chamber and the nozzle.
\end{abstract}

Keywords: jet pump, efficiency, swirling the flow.

\section{INTRODUCTION}

Contrary to the opinion of the primary use of ejection technologies directly for oil and gas production, the industrial use of downhole jet pumps began with the implementation of gas utilization processes, flushing of sand plugs and cleaning the bottom during drilling. In the early 50s of the last century, industrial ejection units were developed for the utilization of low-pressure petroleum gas in the systems of gathering [1] and preparation [2] of well production. Nowadays a significant number of global and regional companies are present in the oilfield services market of petroleum gas utilization.

Sand flushing technology was introduced in the late 50s and early 60s of the last century [3] and was used to clean up shallow wells. In the first half of the 70s, a technology was developed for joint use of coiled tubing and jet pump for cleaning wells. The technology has proven to be so successful that today $30-40 \%$ of coiled tubing operations are associated with sand cleaning of wells [4]. This technology is especially effective for cleaning horizontal wells, the number of which has rapidly increased as a result of the development of "shale" oil fields.

In the 60 s of the 20 th century, a technology was developed to use a jet pump to remove 
objects left during drilling from the bottom of a well.

Further spread of borehole jet pumps is limited by their low energy efficiency: the efficiency of an ejection system, as a rule, does not exceed 35\% [5-8]. The need to improve the energy efficiency of borehole jet pumps is of particular relevance in the implementation of long-term technological operations and, in particular, in the operation of oil wells. One of the ways to optimize the characteristics of ejection systems is to swirl mixed flows in the flow path of jet devices $[9,10]$. Despite a simple design, mechanism of mixing swirling flows is insufficiently studied today.

The purpose of the research, the results of which are presented in this article, is to simulate the characteristics of a jet vortex pump.

Let us determine the influence of design and technological factors on the rotation velocity of fluid particles $\omega$, taking into account that this value is a determining parameter characterizing the process of local swirling of the flow in the flow path of the jet pump. The rotation velocity of the fluid obviously depends on the inclination angle of elements for the direction of the flow $\alpha$, the diameter of the helical trajectory $d$, which is described by fluid particles, and flow rates of the swirling flow $Q$

$$
\omega=f(\alpha, d, Q)
$$

The nature of dependence (1) is determined by the obvious relations (Figure 1)

$$
V=\frac{V_{0}}{\cos \alpha} ; V=\frac{V_{\theta}}{\sin \alpha} ; V_{0}=\frac{4 Q}{\pi d^{2}} ; V_{0}=\omega \frac{d}{2},
$$

where: $V=$ velocity of the swirling flow and $V_{\theta}, V_{0}=$ rotating and axial velocity components.

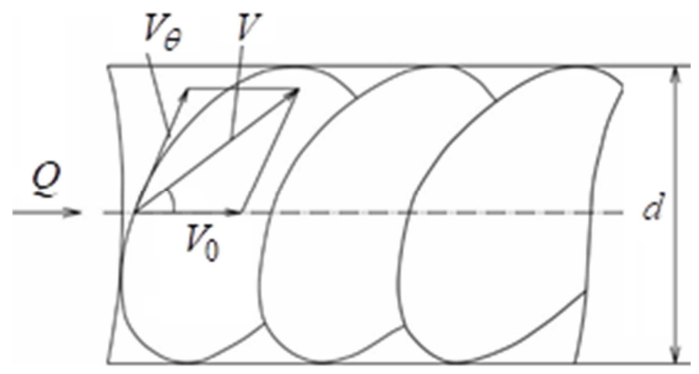

Figure 1 Determination of the swirling velocity

When determining the component $V_{\theta}$, the maximum peripheral velocity is considered.

Considering formula (2), dependence (1) can be represented by the equation as follows

$$
\omega=\frac{8}{\pi} \frac{Q}{d^{3}} \operatorname{tg} \alpha
$$

In addition, we determine the relationship between technological and design parameters in the process of swirling the flow

$$
\frac{V_{\theta}}{V_{0}}=\operatorname{tg} \alpha
$$


Obviously, the above ratio changes from zero at $\alpha=0$ to infinity when the angle approaches $90^{\circ}$ and ceases to exist at $\alpha=90^{\circ}$. The range of swirling flow rate variation is limited by linear dependencies $\omega=f(Q)$ according to equation (3).

Let us determine the pressure distribution in the field of a plane vortex using Gromek interpretation of the Euler integral, which is a special case of the Lagrange equation. Bernoulli's equation for this case cannot be applied, since it can be used exclusively for fluid particles located on the same flow line. For the case of constant potential motion of the fluid, the total energy of a unit mass of the fluid does not depend on time and is constant for all points of the flow

$$
U+\Pi+\frac{V^{2}}{2}=\text { const }
$$

where $U=$ the potential of body forces; $\Pi=$ the potential of pressure forces and $V=$ the flow rate.

Considering the orientation of the jet pump in the well, the vortex axis is placed vertically. This makes it possible to exclude from consideration the action of gravity $U$ $=0$.

Suppose that the vortex is in a calm medium, that is, the velocity at infinity is zero, and the magnitude of the potential of the pressure forces is $\Pi=\Pi_{\infty}$. Therefore, for any point in the vortex field, we can obtain

$$
\Pi-\Pi_{\infty}=\frac{V^{2}}{2}
$$

The fluid velocity can be determined using the concept of velocity circulation in a closed loop $\Gamma$ with a radius $r_{i}$

$$
\Gamma=2 \pi r_{i} V
$$

Then for an incompressible (fluid density $\rho=\mathrm{const}$ ) medium, taking into account that $\Pi=P / \rho, \Pi_{\infty}=\Pi_{\infty} / \rho_{\infty}$, we obtain

$$
P-P_{\infty}=-\frac{\rho \Gamma^{2}}{8 \pi^{2} r_{i}^{2}}
$$

For the convenient practical use, we transform expression (8) considering the relationship between circulation and the angular velocity of fluid particles rotation

$$
\begin{aligned}
& \Gamma=2 \pi r_{i}^{2} \omega \\
& P-P_{\infty}=\rho \frac{\omega^{2} r_{i}^{2}}{2}
\end{aligned}
$$

The pressure at any point in the vortex field is thus less than the pressure in a calm medium, that is, in the pressure field, fluid is sucked in.

Let us analyze the nature of the pressure distribution in the inlet section of the mixing chamber caused by the flow rotation. According to equation (9), the inertial pressure 
component $\rho \omega^{2} r_{i}^{2} / 2$ causes uneven pressure distribution in the inlet section of the mixing chamber, which changes according to a parabolic law. The vertex of the parabola that defines the pressure plot is located on the axis of the jet pump. In the process of swirling the injected flow, a uniform pressure distribution takes place in the section of the operating flow (Figure 2).

It should be noted that for all considered cases, taking into account peculiarities of the jet pump operating process, the pressure of the injected flow exceeds the value of the pressure of operating flow. For low-pressure jet pumps, there is no narrowing of operating flow in the area between the nozzle and the mixing chamber (trajectory A in Figure 2). In the case of operating jet narrowing (trajectory B in Figure 2), which is taken into account in the analysis of operating process of a high-pressure pump, obviously, the operating section decreases, and the area of the injected flow section increases in the inlet section of a mixing chamber.

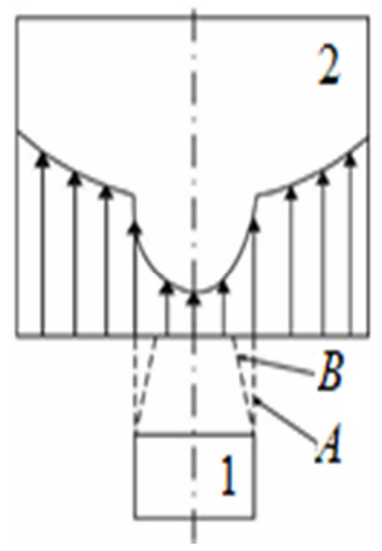

Figure 2 Distribution of pressure in the inlet section of the mixing chamber in the case of injected flow swirling: 1 - a nozzle; 2 - a mixing chamber; $A, B$ - trajectories of operating flow of the low-pressure and high-pressure jet pump, accordingly

When simulating the jet pump process of operation taking into account the flow swirling, we assume that fluid swirling stops in the mixing chamber, as a result of which a uniform distribution of pressures takes place in its outlet section.

The construction of a hydraulic model of the ejection system operation process [11] is based on the use of the equation for conservation of the fluid momentum in the characteristic sections of the flow path of the jet pump (Figure 3)

$$
\begin{aligned}
& \varphi_{2}\left(G_{w} V_{w 1}+G_{s} V_{s 2}\right)-\left(G_{w}+G_{s}\right) V_{m}=\left(P_{m 3}-P_{s 2}^{\prime}\right) f_{s 2}+\left(P_{m 3}-P_{w 1}\right) f_{w 1}= \\
& =P_{m 3} f_{3}-P_{s 2}^{\prime} f_{s 2}-P_{w 1} f_{w 1}
\end{aligned}
$$

where $\varphi_{2}=$ the velocity coefficient for the inlet section of the mixing chamber; $G_{w}, G_{s}$ $=$ mass flow rates of the operating and injected flows; $V_{w 1}, V_{s 2}, V_{m}=$ velocities of the operating flow at the outlet of the nozzle, the injected flow at the inlet to the mixing chamber and the mixed flow at the outlet of the mixing chamber; $P_{m 3}, P_{s 2}^{\prime}, P_{w 1}=$ the pressures of the mixed flow at the outlet of the mixing chamber, injected swirl flow and working flow at the outlet of the nozzle and $f_{s 2}, f_{w 1}, f_{3}=$ a cross-sectional area of the injected flow at the mixing chamber inlet, the nozzle and the mixing chamber outlet. 


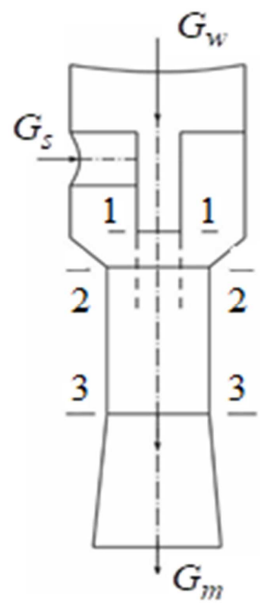

Figure 3 The flow path of the jet pump

The force created by injected flow swirling in the inlet section of the mixing chamber is determined by integrating the unit forces acting on the elementary section area $2 \pi r d r$

$$
\begin{aligned}
& P_{s 2}^{\prime} f_{s 2}=\left(P_{s 2}+\frac{\rho \omega^{2} r^{2}}{2}\right) f_{s 2}=\int_{r_{w}}^{r_{c}}\left(P_{s 2}+\frac{\rho \omega^{2} r^{2}}{2}\right) \times 2 \pi r d r= \\
& =P_{s 2} f_{s 2}+\rho \omega^{2} \frac{\pi}{4}\left(r_{c}^{4}-r_{w}^{4}\right)
\end{aligned}
$$

where $r_{c}, r_{w}=$ the radii of the mixing chamber and the nozzle.

The flow rates in the characteristic sections of the jet pump are determined taking into account the specific density of the operating $v_{w}$, injected $v_{s}$ and mixed $v_{m}$ flows

$$
V_{w_{1}}=\frac{G_{w}}{f_{w_{1}}} v_{w} ; V_{s 2}=\frac{G_{s}}{f_{s 2}} v_{s} ; V_{m}=\frac{G_{w}+G_{s}}{f_{3}} v_{m}
$$

After substituting formulas (11), (12) into equation (10), we obtain

$$
\begin{aligned}
& \varphi_{2}\left(G_{w} \frac{G_{w} v_{w}}{f_{w_{1}}}+G_{s} \frac{G_{s} v_{s}}{f_{s 2}}\right)-\left(G_{w}+G_{s}\right) \frac{G_{w}+G_{s}}{f_{3}} v_{m}= \\
& =P_{m 3} f_{3}-P_{s 2} f_{s 2}-P_{w 1} f_{w_{1}}-\rho \omega^{2} \frac{\pi}{4}\left(r_{c}^{4}-r_{w}^{4}\right)
\end{aligned}
$$

The relationship between the hydrodynamic parameters of the mixing chamber and characteristic cross-sections of a jet pump is carried out using obvious relationships

$$
\begin{aligned}
& P_{m 3}=P_{m}-\frac{\varphi_{3}^{2} V_{m}^{2}}{2 v_{m}}=P_{m}-\frac{\varphi_{3}^{2}\left(G_{w}+G_{s}\right)^{2} v_{m}}{2 f_{3}^{2}} ; \\
& P_{s 2}=P_{s}-\frac{V_{s 2}^{2}}{2 \varphi_{4}^{2} v_{s}}=P_{s}-\frac{G_{s}^{2} v_{s}}{2 \varphi_{4}^{2} f_{s 2}^{2}}
\end{aligned}
$$




$$
P_{w 1}=P_{w}-\frac{V_{w_{1}}^{2}}{2 \varphi_{1}^{2} v_{w}}=P_{w}-\frac{G_{w}^{2} v_{w}}{2 \varphi_{1}^{2} f_{w_{1}}^{2}}
$$

where $P_{m}, P_{s}, P_{w}=$ the pressure of the mixed flow at the outlet of the jet pump and the pressure of the injected and operating flow at the inlet to the jet pump and $\varphi_{1}, \varphi_{3}, \varphi_{4}=$ the velocity factor for the nozzle, mixing chamber outlet and a suction port.

Using formulas (14)-(16) and the concept of the injection coefficient, which is determined by the ratio of injected $Q_{s}$ and operating $Q_{w}$ flow rates $i=Q_{s} / Q_{w}$ after obvious transformations, equation (13) can be written in the form as follows

$$
h=\frac{P_{m}-P_{s}}{P_{w}-P_{s}}=\varphi_{1}^{2} \frac{f_{w_{1}}}{f_{3}} \times A+\frac{\rho \omega^{2} \pi\left(r_{c}^{4}-r_{w}^{4}\right) f_{w 1}}{2 G_{w}^{2} v_{w}}
$$

where

$$
A=2 \varphi_{2}+\left(2 \varphi_{2}-\frac{1}{\varphi_{4}^{2}}\right) \frac{i^{2}}{K_{p}-1} \frac{f_{w 1}}{f_{3}-f_{w}} \frac{v_{s}}{v_{w}}-\left(2-\varphi_{3}^{2}\right)(1+i)^{2} \frac{f_{w 1}}{f_{3}} \frac{v_{m}}{v_{w}}
$$

where $K_{p}=$ the basic geometric parameter of the jet pump, $K_{p}=\left(f_{3} / f_{w 1}\right)$.

Given the obvious transformations

$$
\frac{f_{w 1}}{f_{3}}=\frac{1}{K_{p}} ; \frac{f_{3}-f_{w 1}}{f_{w 1}}=K_{p}^{-1},
$$

we obtain

$$
h=\frac{\varphi_{1}^{2}}{K_{p}}\left[2 \varphi_{2}+\left(2 \varphi_{2}-\frac{1}{\varphi_{4}^{2}}\right) \frac{i^{2}}{K_{p}-1} \frac{v_{s}}{v_{w}}-\left(2-\varphi_{3}^{2}\right) \frac{(1+i)^{2}}{K_{p}} \frac{v_{m}}{v_{w}}\right]+\frac{\varphi_{1}^{2} \rho \omega^{2} \pi\left(r_{c}^{4}-r_{w}^{4}\right) f_{w_{1}}^{2}}{2 G_{w}^{2} v_{w} f_{3}}
$$

Let us transform the component of equation (18), which determines the additional dynamic pressure caused by swirling of the injected flow using the elementary dependences

$$
\frac{G_{w}{ }^{2} v_{w}}{f_{w_{1}}^{2} \rho}=\frac{G_{w}{ }^{2} v_{w}^{2}}{f_{w_{1}}^{2}}=V_{w_{1}}^{2} ; f_{3}=\pi r_{c}^{2} ; f_{w 1}=\pi r_{w}^{2}
$$

Then the component of equation (18), which determines the additional dynamic pressure, takes the form

$$
\frac{\varphi_{1}^{2} \rho \omega^{2} \pi\left(r_{c}^{4}-r_{w}^{4}\right) f_{w_{1}}^{2}}{2 G_{w}^{2} v_{w} f_{3}}=\frac{\varphi_{1}^{2}}{2}\left(\frac{V_{\theta c}^{2}}{V_{w_{1}}^{2}}-\frac{V_{\theta w}^{2}}{V_{w_{1}}^{2} K_{p}}\right)
$$

where the rotating velocities for the mixing chamber $V_{\theta c}$ and the working nozzle $V_{\theta w}$ are determined by the formulas $V_{\theta c}=\omega r_{c} ; V_{\theta w}=\omega r_{w}$. 
Thus, the additional pressure caused by the fluid rotation is determined by the value of the rotating and axial velocities of swirling flows.

For the possibility of practical use, the last component of equation (18) must be converted to an engineering form. Let us determine the form of the equation for calculating the swirl rate of the injected flow. The axial velocity of the injected flow with the flow rate $Q_{s}$ is determined by the formula

$$
V_{0 s}=\frac{4 Q_{s}}{\pi\left(d_{c}^{2}-d_{w}^{2}\right)}
$$

The value of the average rotating velocity of the injected flow can be determined by the formula as follows

$$
V_{\theta s}=\frac{\omega\left(d_{c}+d_{w}\right)}{4}
$$

Then the resulting velocity of injected flow swirling can be determined by the formulas

$$
\begin{aligned}
& V_{s}=\frac{V_{0 s}}{\cos \alpha_{s}}=\frac{4 Q_{s}}{\pi\left(d_{c}^{2}-d_{w}^{2}\right) \cos \alpha_{s}} ; \\
& V_{s}=\frac{V_{\theta s}}{\sin \alpha_{s}}=\frac{\omega\left(d_{c}+d_{w}\right)}{4 \sin \alpha_{s}}
\end{aligned}
$$

where $\alpha_{s}=$ the inclination angle of guiding elements for swirling the injected flow.

The general solution of the system of equations (23), (24) makes it possible to determine the angular velocity of the injected flow

$$
\omega_{s}=\frac{4 Q_{s} \operatorname{tg} \alpha_{s}}{f_{s}\left(1+K_{p}^{0,5}\right) d_{w}}
$$

where $f_{s}=$ the area of injected flow.

Using the obvious dependences

$$
G_{w}^{2} v_{w}^{2}=Q_{w}^{2} ; \pi\left(r_{c}^{2}-r_{w}^{2}\right)=f_{s}
$$

the formula for determining the additional pressure caused by the fluid rotation can be written in the form

$$
\frac{\varphi_{1}^{2} \rho \omega^{2} \pi\left(r_{c}^{4}-r_{w}^{4}\right) f_{w_{1}}^{2}}{2 G_{w}^{2} f_{3} v_{w}}=\frac{\varphi_{1}^{2} \omega^{2} f_{s} f_{w_{1}}^{2}\left(1+K_{p}^{-1}\right)}{2 Q_{w}^{2} \pi}
$$

After substitution of formula (25) into equation (26) and implementation of the substitution

$$
\frac{Q_{s}^{2}}{Q_{w}^{2}}=i^{2} ; \frac{\pi d_{w}^{2}}{4}=f_{w 1}
$$

we obtain a formula for determining the dynamic head in the form as follows 


$$
h_{d}=\frac{2 \varphi_{1}^{2} i^{2} \operatorname{tg}^{2} \alpha_{s}\left(1+K_{p}^{-1}\right)}{\left(1+K_{p}^{0,5}\right)^{2}\left(K_{p}-1\right)}
$$

Taking into account the latter dependence, head characteristics of the jet pump under conditions of injected flow swirling have the following form (equation (18))

$$
h=\frac{\varphi_{1}^{2}}{K_{p}} \times B+\frac{2 \varphi_{1}^{2} i^{2} t^{2} \alpha_{s}\left(1+K_{p}^{-1}\right)}{\left(1+K_{p}^{0,5}\right)^{2}\left(K_{p}-1\right)}
$$

In the case of zero values of the injected flow rate $Q_{s}$ and the value of the injection coefficient $i$, there is no additional dynamic head, and the relative head takes on maximum values and can be determined by the formula

$$
h_{\max }=\frac{\varphi_{1}^{2}}{K_{p}}\left[2 \varphi_{2}-\frac{\left(2-\varphi_{3}^{2}\right)}{K_{p}} \frac{v_{m}}{v_{w}}\right]
$$

The efficiency of using the guiding elements in the flow path of the jet pump can be determined by comparing the value of the relative head obtained for the direct and swirling flows

$$
\Delta h=\frac{h-h_{s}}{h} \times 100 \%
$$

where $h, h_{s}=$ the relative head for swirling and direct injected flows.

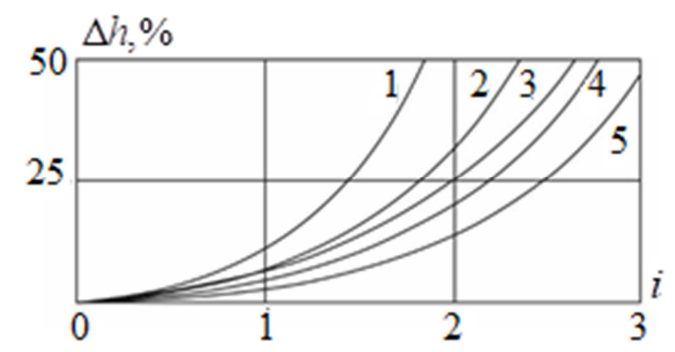

Figure 4 Dependence of the pressure growth on the injection coefficient of the jet pump for different values of the main geometric parameter: $1-K_{p}=4,0 ; 2-K_{p}=5,0 ; 3$ -

$$
K_{p}=6,0 ; 4-K_{p}=7,0 ; 5-K_{p}=8,0
$$

The dependencies $\Delta h=f(i)$ are made using equations (28) and (30) and have an upward character (Figure 4).

The coefficient of efficiency of the jet pump is determined by the ratio

$$
\eta=\frac{h i}{(1-h)}
$$

The value of the relative head $h$ included in equation (31) is calculated by formula (28). The increase in efficiency caused by swirling of the injected flow is determined by the ratio (Figure 5) 


$$
\Delta \eta=\frac{\eta-\eta_{s}}{\eta} \times 100 \%
$$

where $\eta, \eta_{s}=$ the efficiencies for swirling and direct injected flow.

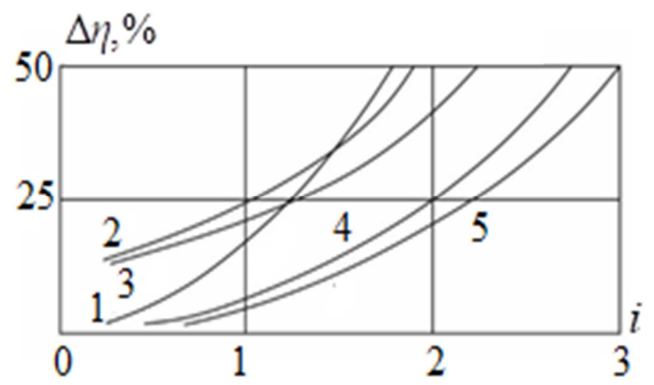

Figure 5 Dependence of efficiency growth on the injection coefficient of a jet pump for different values of the main geometric parameter: $1-K_{p}=4,0 ; 2-K_{p}=5,0 ; 3-K_{p}$

$$
=6,0 ; 4-K_{p}=7,0 ; 5-K_{p}=8,0
$$

Thus, the value of the relative head and the efficiency of the ejection system caused by the swirling of the mixed flows and the value of the injection coefficient of the jet pump are related to each other by a directly proportional nonlinear relationship. The efficiency of swirling mixed flows decreases with an increase in the main geometric parameter of the jet pump.

\section{CONCLUSIONS}

The proposed and theoretically substantiated mechanism of local circulation of mixed flows is based on the use of the law of momentum conservation of fluid in adjacent jets with parabolic pressure distribution. The obtained equations make it possible to additionally take into account the presence of guiding elements in the flow path of the jet pump for swirling the flow and to establish the features of the operating process of vortex ejection systems:

- additional pressure, caused by the rotation of the fluid, is determined by the value of rotating and axial velocities of swirling flows;

- in the case of zero values of the injected flow rate and the value of the injection coefficient, there is no additional dynamic pressure caused by the rotation of the operating medium;

- an increase in the relative pressure and efficiency caused by swirling of the mixed flows, in direct proportion to the flow rate of the injected flow and the value of the injection coefficient of the jet pump;

- an increase in the main geometric parameter of the jet pump reduces the efficiency of swirling the mixed flows.

\section{REFERENCES}

[1] Kolesnik A.P., Polyanskij A.P., Eroshkin S.G., On the use of ejectors for transport of associated oil gas, Oil industry, Russian, vol. 3, pp 79-84, 1955. 
[2] Yusupov Ya.Yu., Scheme of collection and transportation of oil and gas using a gas jet ejector, Oil industry, Russian, vol. 7, pp 45-48, 1957.

[3] Bogdanov A.A., Pomazkova Z.S., Jet devices for washing sand plugs in wells, Russian, 1960.

[4] Yateem K.S., Qahtani H.B., Fill cleanout operations in offshore Saudi Arabian Fields: Case histories toward Improving Economic 1 and operational Logistics, International Petroleum Technology Conference, Beijing, China, 26-28 March, 2013, pp 1954-1971, IPTC-16677-MS, https://doi .org/10.2523/IPTC-16677-MS.

[5] Gugulothy S.K., Manchikatla S., Experimental and performance analysis of single nozzle jet pump with various mixing tubes, International Journal of Recent advances in Mechanical Engineering (IJMECH), Australia, vol. 3, issue 4, pp 119-133, 2014.

[6] Ismagilov A.R., Spiridonov E.K., Operational process and characteristics of liquidgas jet pumps with the ejected vapor-gas medium, Procedia Engineering, United Kingdom, vol. 150, pp 247-253, 2016.

[7] Sheha A.A.A., Nasr M., Hosien M.A., Wahba E.M., Computational and experimental study on the water-jet pump performance, Journal of Applied Fluid Mechanics, Iran, vol. 11, issue 4, pp 1013-1020, 2018.

[8] Kryzhanivskyi E.I., Panevnyk D.A., Improving use efficiency above-bit jet pumps, Socar proceeding, Azerbaijan, vol. 2, pp 112-118, 2020.

[9] Kawaguty K., Ueki H., Akamine S., Umeoka T., Experimental research on air mixed jet pump for sea water purification, Proceeding of the Sixth International Offshore and Polar Engineering Conference, USA, 26-31 May, 1996, pp 134-142, 1996.

[10] Wittrisch C., Trapy J., Hydraulic jet pumps. Modeling and improvements, Offshore Mediterranean Conference and Exibition, Italy, 26-28 March 2003, pp. 9, 2003

[11] Sokolov E.Ya., Zinger N.M., Inkjet devices, Russian, 1989. 